\title{
Membership, binarity, and rotation of F-G-K stars in the open cluster Blanco $1^{\star, \star \star}$
}

\author{
J.-C. Mermilliod ${ }^{1}$, I. Platais ${ }^{2}$, D. J. James ${ }^{3,4}$, M. Grenon ${ }^{5}$, and P. A. Cargile ${ }^{3}$ \\ ${ }^{1}$ Laboratoire d'Astrophysique, École polytechnique fédérale de Lausanne (EPFL), Observatoire, 1290 Sauverny, Switzerland \\ e-mail: Jean-Claude.Mermilliod@epfl.ch \\ 2 Department of Physics and Astronomy, Johns Hopkins University, 3400 North Charles Street, Baltimore, MD 21218, USA \\ Department of Physics \& Astronomy, Box 1807 Station B, Vanderbilt University, Nashville, TN 37235, USA \\ 4 Department of Physics, Fisk University, 1000 17th Avenue North, Nashville, TN 37208, USA \\ 5 Observatoire de Genève, 1290 Sauverny, Switzerland
}

Received 15 November 2007 / Accepted 25 April 2008

\begin{abstract}
Context. The nearby open cluster Blanco 1 is of considerable astrophysical interest for formation and evolution studies of open clusters because it is the third highest Galactic latitude cluster known. It has been observed often, but so far no definitive and comprehensive membership determination is readily available.

Aims. An observing programme was carried out to study the stellar population of Blanco 1, and especially the membership and binary frequency of the F5-K0 dwarfs.

Methods. We obtained radial-velocities with the CORAVEL spectrograph in the field of Blanco 1 for a sample of 148 F-G-K candidate stars in the magnitude range $10<V<14$. New proper motions and $U B V I$ CCD photometric data from two extensive surveys were obtained independently and are used to establish reliable cluster membership assignments in concert with radial-velocity data. Results. The membership of 68 stars is confirmed on the basis of proper motion, radial velocity, and photometric criteria. Fourteen spectroscopic- and suspected binaries (2 SB2s, 9 SB1s, 3 SB?) have been discovered among the confirmed members. Thirteen additional stars are located above the main sequence or close to the binary ridge, with radial velocities and proper motions supporting their membership. These are probable binaries with wide separations. Nine binaries (7 SB1 and 2 SB2) were detected among the field stars. The spectroscopic binary frequency among members is 20\% (14/68); however, the overall binary rate reaches $40 \%$ (27/68) if one includes the photometric binaries. The cluster mean heliocentric radial velocity is $+5.53 \pm 0.11 \mathrm{~km} \mathrm{~s}^{-1}$ based on the most reliable 49 members. The $V \sin i$ distribution is similar to that of the Pleiades, confirming the age similarities between the two clusters.

Conclusions. This study clearly demonstrates that, in spite of the cluster's high Galactic latitude, three membership criteria - radial velocity, proper motion, and photometry - are necessary for performing a reliable membership selection. Furthermore, even with accurate and extensive data, ambiguous cases still remain.
\end{abstract}

Key words. Galaxy: open clusters and associations: general - Galaxy: open clusters and associations: individual: Zeta Scl techniques: radial velocities - techniques: photometric

\section{Introduction}

Blanco 1 (C0001-302) is a nearby, young ( 60-100 Myr) open cluster seen in the direction of the south Galactic pole at $\alpha=$ $0^{\mathrm{h}} 04^{\mathrm{m}} 1, \delta=-29^{\circ} 50^{\prime}(\mathrm{J} 2000)$, at a distance of about $250 \mathrm{pc}$ and at high Galactic latitude $\left(b=-79^{\mathrm{d}} \cdot 26\right)$. The first extensive investigations were photometric. de Epstein \& Epstein (1985) published photographic $u v b y$ data and $B-V$ indices computed from uvby for 261 stars. Westerlund et al. (1988) obtained photoelectric observations for 130 stars, mostly brighter than $V=12$, on the $U B V \beta$ system, although nearly a half of their observed stars also have uvby data. The photographic survey of de Epstein \& Epstein has been used as a reference for the star numbering in

* Based on observations collected with the Danish 1.54-m and the Swiss telescopes at the European Southern Observatory, La Silla, Chile, and with the old YALO 1-m telescope at the Cerro Tololo InterAmerican Observatory, Chile.

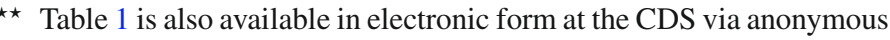
ftp to cdsarc.u-strasbg. fr $(130.79 .128 .5)$ or via http://cdsweb.u-strasbg.fr/cgi-bin/qcat?]/A+A/485/95 several subsequent papers because it extends to fainter magnitudes $(V<16)$. In particular, the de Epstein \& Epstein stars were observed by Panagi et al. (1994) and Panagi \& O'Dell (1997) to study their $\mathrm{H} \alpha$ emission intensities and to measure the equivalent width of the resonance line of lithium at $6708 \AA$, in an attempt to derive the cluster's age by comparison to similar observations for other young open clusters.

A rather high metal abundance, $[\mathrm{Fe} / \mathrm{H}]=+0.23$, was derived by Edvardsson et al. (1995) for the cluster. This value was revised to a nearly solar value, $[\mathrm{Fe} / \mathrm{H}]=+0.04$, by Ford et al. (2005). These determinations contrast the earlier value of $[\mathrm{Me} / \mathrm{H}]=-0.17$ derived from uvby photometric data by Westerlund et al. (1988). The main difference between the spectroscopic determinations seems to be caused by a significantly higher-temperature scale adopted by Edvardsson et al. (1995).

Blanco 1 was also observed in X-rays using the ROSAT and XMM-Newton satellites. Micela et al. (1999) detected 132 sources in the ROSAT field of view $\left(40^{\prime} \times 40^{\prime}\right)$, of which only 42 were identified with known cluster members. 
Pillitteri et al. (2003) studied the X-ray luminosity function on the basis of these data. Membership of optical counterparts to $\mathrm{X}$-ray sources was estimated from the GSC-II proper motions and $B R$ photometry. The X-ray detections allowed the identification of late-K and $\mathrm{M}$ Blanco 1 candidate stars, whose cluster membership is supported by the $B R$ photometric data and proper motions.

Pillitteri et al. (2004) detected 190 sources in the XMM-Newton EPIC camera field (a field-of-view of $30^{\prime} \times 30^{\prime}$ ), of which 36 were identified with known members. Additional optical identifications were found for another 90 X-ray sources in large survey catalogues, while some 64 sources remained unidentified. Finally, Pillitteri et al. (2005) studied the variability of these stars and published the light curves of $14 \mathrm{X}$-ray variable cluster members.

Recently, Moraux et al. (2007) surveyed Blanco 1 using $I Z K_{\mathrm{s}}$ photometry to search for very low mass stars and brown dwarfs. They catalogued about 300 candidate members, of which 30-40 could be brown dwarfs. Follow-up spectroscopic observations should indicate which of these candidates are bona fide members.

It is difficult to estimate precisely the age of Blanco 1 by the usual method of isochrone fitting because of the lack of evolved stars on the upper main sequence. However, the comparison of $\mathrm{H} \alpha$ emitters, the lithium trend as a function of $B-V$, and the $\mathrm{X}$-ray luminosity distribution for the Pleiades and Blanco 1 indicate that both clusters are about the same age (Ford et al. 2005).

So far only a handful of radial velocities for Blanco 1 were published - by Edvardsson et al. (1995) using the CORAVEL and CASPEC spectrographs, and by Jeffries \& James (1999) using the UCLES spectrograph. The detailed data may be found in the open cluster database (WEBDA at http://www. univie. ac.at/webda/). A proper motion study of the cluster was reported by Hawkins \& Favata (1998), but the detailed data have not been published so far. Only limited information on the GSC-II proper motions is provided in Pillitteri et al. (2003) paper.

To overcome this lack of fundamental membership data for Blanco 1, we determined reliable cluster membership assignments for stars in the vicinity of Blanco 1 by exploiting new observations in kinematic and astrometric space. First, we performed a radial-velocity survey of candidate cluster members known from the photometric studies of de Epstein \& Epstein (1985) and Westerlund et al. (1988), as well as candidate stars selected from the Selected Area 140 region (Brück 1935) to establish kinematic membership and to study binarity. Second, we determined astrometric membership probabilities for stars in our survey by using a new catalogue of long time base-line proper motions in the region of Blanco 1 (Platais et al. 2008). New probable cluster members selected from the first version of the Southern Proper Motion programme (SPM) were later added to our observing sample - which gives a total of 148 stars.

In this paper, we report the results of our kinematic and proper-motion catalogues for stars in the vicinity of Blanco 1, as well as new UBVI CCD photometry for stars not included in Westerlund's (1988) photoelectric-photometry survey. We assign membership of Blanco 1 to those stars lying on the apparent cluster $B-V$ and $V-I$ main sequences, which also share common properties in radial-velocity space and in the proper-motion vector-point diagram. For the bona fide members, we establish the mean proper motion and mean radial velocity of the cluster, and determine its binary fraction. A brief discussion of the angular momentum of the solar-type stars in Blanco 1 is also presented.

\section{Observations}

The initial selection of stars for the CORAVEL observing programme was based on the photometric studies of de Epstein \& Epstein (1985) which covers a circular area of 1.5 in radius. We selected the stars located near the main sequence and on the red side of the Böhm-Vitense gap, at spectral type F5, and brighter than $B=15$. We also searched the Selected Area 140 for candidates on the basis of their magnitudes and spectral types. This portion of the sample has many stars in common with the list published later by Westerlund et al. (1988), who also added several new objects. Finally, Platais proposed a list of additional candidates selected from the Southern Proper Motion programme (SPM). Only a subset of these new candidates could actually be measured using CORAVEL because the limiting magnitude of the instrument is at $B=15$. The CCD data listed in Table 1 show that we succeeded in observing a few stars slightly fainter than this limit during the best nights. The final sample contained a total of 148 stars distributed in a circle of 1.5 in radius, which is close to the apparent stability radius of 1.8 (see Sect. 4.7).

\subsection{Radial-velocity observing campaigns}

The radial-velocity observations were obtained with the photoelectric scanner CORAVEL (Baranne et al. 1979) on the Danish 1.54-m telescope at ESO, La Silla, Chile, over the course of several observing runs. The very first observations were made in July 1983 and June 1984, but the bulk of observations were obtained in August 1985. Additional radial velocities were obtained in August 1986 and July 1991. The last measurements were made in July 1996.

The radial velocities are reduced to the system defined by Udry et al. (1999), calibrated with high-precision data from the ELODIE spectrograph (Baranne et al. 1996). A total of 518 observations were obtained for 148 programme stars. On average, 4 to 6 observations were obtained for constant-velocity targets.

Projected rotational velocities $(V \sin i)$ were derived from the width of the correlation functions, following the outline by Benz \& Mayor (1984).

The individual radial velocities, with Julian dates of observation, will be published in a comprehensive catalogue of more than 7800 measurements for 1264 dwarfs in nearby open clusters (Mermilliod et al. 2008). Meanwhile, the data for Blanco 1 can be obtained from the first author (JCM).

\section{2. $C C D$ observations}

The $U B V I_{\mathrm{c}}$ CCD photometry for Blanco 1 was obtained using the STA $4064 \times 4064$ CCD camera - the Y4kCAM, attached to the $1.0 \mathrm{~m}$ telescope at the Cerro Tololo Interamerican Observatory, Chile (CTIO). This camera has $15 \mu \mathrm{m}$ pixels, yielding an effective field-of-view of $19 ! 3 \times 19 ! 3$.

Two observing visits of Blanco 1 were carried out, one during 15-17th September, 2005 and one during the night of 30th June, 2007. The first study covered the central $1.6 \times 1.3$ of the cluster, based on the WEBDA database's centre coordinates, while the return visit was used to measure photometry for about 15 outlying stars not covered during the original survey. During each observing run, exposures times were set to be $35,20,10$ and $7 \mathrm{~s}$ in the $U, B, V$ and $I_{\mathrm{c}}$ filters respectively.

For each night, instrumental-magnitude zero points, colourtransformation coefficients and extinction coefficients were calculated by comparison to a minimum of 50 photometric 
standard stars, whose standard magnitudes are available in Landolt (1992). External errors in transforming observed, instrumental magnitudes, onto the standard system are $<2 \%$ over the magnitude and colour range of Blanco 1 targets. A full description of the data reduction and analysis is presented in James et al. (2008), including a complete photometric catalogue in $U B V I_{\mathrm{c}}$ bands.

Only $V$ magnitudes and $B-V$ indices are presented in the printed version of Table 1, in the interest of saving space, however both $U-B$ and $V-I$ data are given in electronic form at the CDS.

\subsection{Proper motions and membership probabilities}

The astrometric proper motions are deduced from 32 sets of photographic plates and CCD frames almost all of them obtained with the $51 \mathrm{~cm}$ double astrograph $\left(\right.$ scale $\left.=55^{\prime \prime} .1 \mathrm{~mm}^{-1}\right)$ at Cesco Observatory in El Leoncito, Argentina. These observations span 40 years, ending in September 2007. There is a mix of visual-bandpass plates and blue plates. Part of photographic observations were obtained with the objective wiregrating, which produces secondary images allowing the correction of magnitude-related systematic errors in the positions, and with two exposures (long and short) on the same plate, thus providing a dynamical range of $\sim 12 \mathrm{mag}$. All CCD observations were obtained with a PixelVision $4 \mathrm{~K} \times 4 \mathrm{~K}$ visual $\mathrm{CCD}$ camera covering nearly a full square degree (Casetti-Dinescu et al. 2007). For the sake of a wider dynamical range, the CCD observations were also taken with the wire-grating.

All stars down to $B_{J}=16$ were measured in a circular area with a 3.8 diameter, centered on $\mathrm{RA}=0^{\mathrm{h}} 0^{\mathrm{m}} 5^{\mathrm{s}}$ and $\mathrm{Dec}=-30^{\circ} 5^{\prime}$ (J2000). These 4440 stars were measured with the Yale PDS microdensitometer in object-by-object mode on all available $17 \times$ 17 in SPM plates (SPM 455 according to the field nomenclature in Platais et al. 1998). A set of five $5 \times 7$ in blue plates used by de Epstein \& Epstein (1985) in their study of Blanco 1 was digitized with the Hubble Space Telescope Science Institute's GAMMA multi-channel scanning microdensitometer (Laidler et al. 1994). Proper motions and positions are calculated using a variant of the central plate-overlap method (e.g., Herbig \& Jones 1981) and the UCAC2 catalogue (Zacharias et al. 2004) as a reference frame and also as an additional set of positions. The precision of proper motions for stars with optimal image properties is $0.3 \mathrm{mas}_{\mathrm{yr}^{-1}}$. The formal proper-motion membership probabilities $P_{\mu}$ were calculated using the probability definition formulated by Vasilevskis et al. (1958). We used the so-called local sample method, which works very well in sparse open clusters (Platais et al. 2008). In this method, for each target star a local sample of stars is selected to represent closely the target's photometric properties. The external proper-motion dispersion for

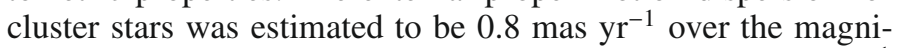
tude range $V=8-14$ and gradually increasing to $\sim 1.5$ mas yr$^{-1}$ at $V=17$. There is good agreement between this estimate and the individual proper-motion error calculation.

The adopted centroid of the proper-motion distribution for cluster stars is $\mu_{\alpha} \cos \delta=+21.8$ and $\mu_{\delta}=+3.1$ mas $\mathrm{yr}^{-1}$. It is close to the Hipparcos mean absolute proper motion for Blanco 1 (Robichon et al. 1999). However, our mean proper motion for Blanco 1 cannot be considered as an independent measure of its absolute proper motion because we used the UCAC2 as an initial reference frame.

The final astrometric catalogue contains 6300 objects. A full description of the data reduction and analysis will be presented in Platais et al. (2008).

\subsection{Results}

In Table 1, we present CORAVEL mean radial velocities, photometry and proper motions for all 148 stars in our sample. We adopt the numbering system used in WEBDA, which is based on Westerlund et al. (1988) list up to number 130; we added new candidate stars under numbers 330 to 361 . Numbers from 1013 to 1258 are based on de Epstein \& Epstein (1985) list (their number +1000$)$. The second and third columns reproduce the original numbers from the Selected Area 140 (Brück 1935) and of de Epstein \& Epstein (1985). The J2000 celestial coordinates come from Platais et al. (2008).

The last column provides remarks on membership and duplicity. NM stands for non-member and M: indicates which stars are members according to radial velocity and photometry but with low astrometric membership probability. In the case of a confirmed or suspected duplicity "SB" stands for spectroscopic binary (members and non-members) while "dbl" designates radial velocity and proper motion members that appear to be photometric double stars.

\section{Membership}

The availability of three major parameters for assessing probable cluster membership, i.e., photometry, radial velocities and proper motions, allows us to discuss the cluster membership in considerable detail.

\subsection{Radial velocities}

A histogram of the mean radial velocities (Fig. 1) shows a sharp peak around $V_{\mathrm{r}} \sim+6 \mathrm{~km} \mathrm{~s}^{-1}-$ a clear sign of kinematic cluster members.

However, due the presence of undetected binaries or marginally variable stars, it is difficult to strictly apply a $3 \sigma$ criterion (in the present case corresponding to $2.1 \mathrm{~km} \mathrm{~s}^{-1}$ ) for membership selection from radial velocities. Instead, we considered all stars in the range $+2<V_{\mathrm{r}}<+10 \mathrm{~km} \mathrm{~s}^{-1}$ to be candidate cluster members and checked their membership by using in addition astrometric and photometric criteria. For suspected binaries, these two membership criteria were considered first.

Accordingly, stars with radial velocities outside the chosen membership range are considered as non-members, if their multi-epoch radial velocities are constant $\left(P\left(\chi^{2}\right)>0.005\right)$, and their proper motion and photometric status also indicates that they are field stars.

Twelve stars, viz, 8, 92, 117, 119, 341, 343, 344, 345, 1122, $1187,1214,1239$, have radial velocities compatible with membership, i.e., $+2<V_{\mathrm{r}}<+10 \mathrm{~km} \mathrm{~s}^{-1}$, but are either located away from the main-sequence (hereafter MS) locus and/or have proper motions significantly different from the cluster mean motion. They have a membership probability equal to zero.

\subsection{Proper motions}

According to our new proper-motion catalogue, the separation between the Blanco 1 open cluster and the Galactic field is convincing (Fig. 2). There is a very good agreement between the proper-motion membership probabilities and the membership based on radial velocities, which proves the reliability of kinematic data for selecting the member stars in Blanco 1 (see Fig. 4).

Four stars $(108,338,351,358)$ have zero proper-motion membership probability but have radial velocities close to the 
Table 1. Mean radial and rotational velocities for stars in Blanco 1.

\begin{tabular}{|c|c|c|c|c|c|c|c|c|c|c|c|c|c|c|c|c|c|}
\hline $\begin{array}{l}\text { No } \\
\text { (1) }\end{array}$ & $\begin{array}{l}\text { SA } \\
(2)\end{array}$ & $\begin{array}{l}\text { ZS } \\
\text { (3) }\end{array}$ & $\begin{array}{l}\text { RA } \\
(4)\end{array}$ & $\begin{array}{l}\text { Dec } \\
(5)\end{array}$ & $\begin{array}{c}V \\
(6)\end{array}$ & $\begin{array}{c}B-V \\
(7)\end{array}$ & $\begin{array}{c}V_{\mathrm{r}} \\
(8)\end{array}$ & $\begin{array}{c}\epsilon \\
(9)\end{array}$ & $\begin{array}{r}N \\
(10)\end{array}$ & $\begin{array}{c}\Delta T \\
(11)\end{array}$ & $\begin{array}{c}P\left(\chi^{2}\right) \\
(12)\end{array}$ & $\begin{array}{c}V \sin i \\
(13)\end{array}$ & $\begin{array}{r}e v s \\
(14)\end{array}$ & $\begin{array}{c}\mu_{\alpha \cos \delta} \delta \\
(15)\end{array}$ & $\begin{array}{c}\mu_{\delta} \\
(16)\end{array}$ & $\begin{array}{r}P_{\mu} \\
(17)\end{array}$ & $\begin{array}{l}\text { Notes } \\
(18)\end{array}$ \\
\hline 8 & 66 & & 235926.73 & $-2851 \quad 13.9$ & 10.10 & 0.48 & +4.74 & 0.25 & 6 & 4737 & 0.675 & 14.9 & 0.7 & 25.6 & -7.8 & 0 & NM \\
\hline 15 & & 3 & 000011.33 & -295042.5 & 11.49 & 0.74 & -8.32 & 1.66 & 5 & 350 & 0.000 & 4.1 & 1.5 & 11.4 & -74.5 & 0 & NM, SB \\
\hline 16 & 85 & 9 & 000012.04 & -302050.4 & 10.96 & 0.57 & -6.08 & 0.20 & 5 & 3989 & 0.930 & 6.0 & 1.0 & -0.4 & -31.8 & 0 & NM \\
\hline 18 & 91 & & 000035.38 & -291548.3 & 10.97 & 0.41 & +11.42 & 0.56 & 1 & & & 6.2 & 2.9 & 14.6 & -10.2 & 0 & NM \\
\hline 19 & & & 000045.02 & -290630.2 & 12.32 & 0.88 & +6.31 & 0.36 & 5 & 3971 & 0.008 & 1.0 & 1.0 & 23.9 & 4.4 & 27 & SB2? \\
\hline 20 & 97 & & 000046.60 & -290805.6 & 11.03 & 0.60 & +6.63 & 0.16 & 6 & 3985 & 0.613 & 4.8 & 0.9 & 21.0 & 3.7 & 92 & $\mathrm{dbl}$ \\
\hline 21 & 98 & & 000049.68 & -292127.3 & 11.78 & 0.51 & -23.64 & 0.39 & 2 & 11 & 0.479 & 5.7 & 2.1 & 20.7 & 5.2 & 25 & NM \\
\hline 28 & 116 & 26 & 000124.48 & -303858.3 & 10.50 & 0.40 & +5.20 & 0.23 & 6 & 4401 & 0.611 & 9.0 & 1.1 & 22.4 & 3.6 & 95 & \\
\hline 31 & 127 & & 000142.37 & -290833.6 & 11.17 & 0.64 & -19.56 & 0.24 & 4 & 3987 & 0.402 & 3.4 & 1.9 & 61.6 & -50.2 & 0 & NM \\
\hline 33 & 133 & & 000149.01 & -29 2301.4 & 11.19 & 0.67 & +12.95 & 0.20 & 5 & 3987 & 0.685 & 4.2 & 1.3 & 64.5 & 9.7 & 0 & NM \\
\hline 34 & 132 & & 000157.46 & -2907 20.3 & 11.95 & 0.79 & -74.06 & 1.62 & 3 & 353 & 0.000 & 4.6 & 2.2 & 84.8 & -22.3 & 0 & NM, SB \\
\hline 37 & 139 & 49 & 000211.90 & -30 1502.8 & 9.89 & 0.46 & -5.77 & 2.55 & 2 & 680 & 0.309 & 66.8 & 6.7 & 20.8 & 3.5 & 91 & $\mathrm{dbl}$ \\
\hline 38 & 141 & 48 & 000221.64 & -300821.6 & 10.72 & 0.48 & +2.53 & 1.71 & 3 & 4406 & 0.794 & 69.0 & 15.2 & 20.7 & 3.3 & 90 & \\
\hline 39 & & 57 & 000219.76 & -294556.2 & 11.69 & 0.62 & +24.46 & 0.24 & 4 & 3987 & 0.776 & 1.9 & 1.0 & -57.8 & -39.3 & 0 & NM \\
\hline 41 & & 55 & 000220.78 & -294750.4 & 10.73 & 0.56 & -9.98 & 0.28 & 4 & 3977 & 0.346 & 8.9 & 1.1 & 31.4 & 8.2 & 0 & NM \\
\hline 44 & 145 & & 000225.11 & -290525.9 & 11.09 & 0.49 & +37.24 & 0.27 & 6 & 3991 & 0.303 & 9.7 & 1.1 & 15.1 & -23.2 & 0 & NM \\
\hline 45 & 146 & & 000227.24 & -294653.6 & 11.17 & 0.53 & -0.46 & 0.28 & 4 & 4764 & 0.849 & 10.0 & 1.0 & 16.4 & -1.5 & 0 & NM \\
\hline 47 & 149 & & 000239.24 & -290430.9 & 10.23 & 0.38 & +3.94 & 1.70 & 5 & 3981 & 0.002 & 33.5 & 3.4 & 20.0 & 4.3 & 38 & SB? \\
\hline 48 & & & 000243.93 & -292707.9 & 12.15 & 0.60 & +18.94 & 0.26 & 5 & 3992 & 0.883 & 4.0 & 1.9 & 57.8 & -6.4 & 0 & NM \\
\hline 50 & 157 & 67 & 000252.35 & -301958.1 & 11.86 & 0.71 & +29.53 & 0.57 & 6 & 4765 & 0.000 & 4.4 & 1.4 & -23.7 & -13.8 & 0 & NM \\
\hline 52 & & & 000306.52 & -292144.0 & 11.64 & 0.60 & +5.44 & 0.22 & 5 & 3982 & 0.517 & 9.1 & 0.9 & 19.2 & 3.5 & 8 & \\
\hline 53 & 165 & 84 & 000310.82 & -301048.9 & 11.32 & 0.56 & +4.71 & 0.59 & 5 & 3989 & 0.358 & 24.0 & 2.4 & 23.2 & 3.9 & 82 & \\
\hline 54 & 166 & 82 & 000314.96 & -301832.3 & 10.35 & 0.43 & -10.89 & 1.66 & 4 & 1103 & 0.203 & 65.9 & 6.6 & 31.9 & 2.8 & 0 & NM \\
\hline 55 & 164 & & 000316.03 & -292000.5 & 10.67 & 0.62 & +14.69 & 0.68 & 5 & 2168 & 0.000 & 3.9 & 1.3 & 127.3 & -27.9 & 0 & NM, SB \\
\hline 56 & 169 & 91 & 00032 & -294922.8 & 11.30 & 0.57 & +6.10 & 0.40 & 6 & 3986 & 0.034 & 12.8 & 0.8 & 22.1 & 2.5 & 95 & \\
\hline 57 & 172 & 96 & 00032 & $-3001 \quad 10.5$ & 10.38 & 0.42 & -30.85 & 3.92 & 2 & 2875 & 0.000 & 21.8 & 2.2 & 20.8 & 3.5 & 92 & M:, SB \\
\hline 58 & 171 & 90 & 000324.39 & -294849.4 & 10.62 & 0.50 & +1.43 & 2.24 & 5 & 3984 & 0.020 & 66.0 & 15.0 & 20.7 & 3.5 & 90 & \\
\hline 60 & 175 & 101 & 00032 & -30 1927.2 & 10.61 & 0.44 & +5.20 & 0.37 & 6 & 4759 & 0.965 & 19.1 & 0.9 & 22.1 & 3.0 & 97 & \\
\hline 63 & 176 & 99 & 000333.60 & -302842.0 & 10.62 & 0.45 & +3.73 & 0.19 & 7 & 4759 & 0.355 & 6.0 & 0.9 & 21.6 & 2.7 & 96 & \\
\hline 64 & 447 & 102 & 000333.69 & -301544.0 & 12.50 & 0.77 & +5.65 & 0.23 & 5 & 3987 & 0.453 & 7.4 & 1.1 & 21.7 & 2.6 & 94 & \\
\hline 68 & 188 & 107 & 000350.17 & -300355.7 & 11.04 & 0.54 & +5.18 & 0.40 & 6 & 4398 & 0.012 & 11.5 & 1.0 & 22.0 & 2.9 & 97 & SB2 \\
\hline 70 & 191 & 110 & 000400.02 & -301915.8 & 11.12 & 0.56 & +5.05 & 0.92 & 6 & 4398 & 0.000 & 15.7 & 0.9 & 23.2 & 3.1 & 89 & SB \\
\hline 73 & 198 & 114 & 000416.48 & -294452.9 & 10.81 & 0.56 & +16.35 & 2.30 & 10 & 3993 & 0.000 & 3.9 & 1.1 & 61.6 & 19.9 & 0 & NM, SB \\
\hline 74 & 202 & 123 & 000418.75 & -294932.5 & 10.52 & 0.45 & +2.08 & 2.02 & 3 & 3987 & 0.813 & 31.7 & 6.7 & 21.3 & 3.5 & 95 & \\
\hline 77 & 200 & & 000427.49 & -284346.7 & 10.90 & 0.48 & -4.86 & 0.21 & 6 & 3989 & 0.900 & 7.6 & 0.9 & 6.3 & -8.7 & 0 & NM \\
\hline 79 & & 129 & 000431.67 & -301441.7 & 11.68 & 0.61 & +5.69 & 0.36 & 5 & 3985 & 0.055 & 7.1 & 1.1 & 21.8 & 1.9 & 87 & \\
\hline 80 & & 126 & 00043. & -293828.1 & 11.04 & 0.5 & +5.64 & 0.24 & 5 & 3989 & 0.608 & 8.7 & 1.0 & 22.1 & 3.1 & 96 & \\
\hline 84 & 210 & & 00044 & -290943.6 & 11.19 & 0. & +1.52 & 0.26 & 5 & 3985 & 0.340 & 7.3 & 1.1 & -10.3 & -21.4 & 0 & NM \\
\hline 85 & 214 & 134 & 000449.21 & -300052.8 & 11.14 & 0.54 & +7.05 & 2.11 & 4 & 3982 & 0.019 & 54.0 & 9.4 & 23.4 & 5.0 & 29 & \\
\hline 89 & 219 & & 00045 & -293347.8 & 11.55 & 0.59 & +5.37 & 0.34 & 5 & 3589 & 0.963 & 19.0 & 0.9 & 21.8 & 2.2 & 93 & \\
\hline 91 & 446 & 138 & 000458.84 & -300941.6 & 11.47 & 0.58 & +5.91 & 0.33 & 6 & 3982 & 0.929 & 18.3 & 0.9 & 22.4 & 2.6 & 95 & \\
\hline 92 & 223 & & 000507.25 & -29 1309.8 & 11.33 & 0.44 & +3.47 & 0.27 & 5 & 3989 & 0.373 & 9.4 & 1.0 & 20.5 & 15.3 & 0 & NM \\
\hline 93 & 228 & 146 & 000516.32 & -294039.6 & 11.48 & 0.61 & -29.38 & 0.23 & 4 & 3987 & 0.824 & 2.3 & 1.8 & -29.7 & -22.6 & 0 & NM \\
\hline 95 & 227 & & 000518.40 & -293005.6 & 11.43 & 0.68 & +10.57 & 6.90 & 3 & 3982 & 0.000 & 22.1 & 22.2 & 21.5 & 2.6 & 95 & SB \\
\hline 98 & 232 & 159 & 000529.26 & -300654.4 & 11.33 & 0.70 & +14.72 & 3.86 & 5 & 3984 & 0.000 & 1.7 & 1.6 & 52.3 & -43.8 & 0 & NM, SB \\
\hline 99 & 231 & 160 & 000530.94 & -295308.1 & 11.26 & 0.55 & +5.27 & 0.31 & 5 & 4401 & 0.860 & 15.3 & 1.0 & 21.7 & 3.9 & 94 & \\
\hline 100 & & 167 & 000535.08 & -300210.0 & 12.07 & 0.73 & +27.09 & 0.24 & 4 & 3988 & 0.729 & 0.5 & 3.0 & -7.0 & 2.7 & 0 & NM \\
\hline 107 & 239 & 171 & 000554.71 & -300438.8 & 10.67 & 0.50 & +15.05 & 0.22 & 6 & 3988 & 0.933 & 9.7 & 0.8 & -41.6 & 10.1 & 0 & NM \\
\hline 108 & 238 & & 000556.40 & -285610.7 & 11.45 & 0.56 & +6.14 & 0.45 & 5 & 3989 & 0.091 & 16.5 & 0.8 & 18.3 & 1.6 & 0 & M: \\
\hline 110 & 245 & 176 & 000559.05 & -293904.7 & 11.65 & 0.61 & +6.07 & 0.30 & 5 & 3985 & 0.962 & 15.0 & 0.9 & 22.1 & 3.9 & 94 & \\
\hline 112 & 251 & 186 & 000617.20 & -294222.7 & 12.22 & 0.7 & -0.55 & 2.90 & 6 & 3990 & 0.0 & 3.2 & 1.7 & 32.6 & 0.5 & 0 & NM, SB \\
\hline 113 & 252 & 182 & 00061 & -300557.1 & 11.72 & 0.6 & +5.53 & 0.22 & 6 & 3991 & 0.3 & 8.0 & 0.9 & 21.7 & 3.0 & 96 & \\
\hline 116 & 258 & 189 & 000628.65 & -301749.0 & 11.61 & 0.6 & -32.65 & 13.10 & 8 & 17 & 0.000 & 13.9 & 0.8 & 46.5 & 13.2 & 0 & $\mathrm{NM}, \mathrm{S}$ \\
\hline 117 & 260 & 190 & 000639.34 & -304004.5 & 11.13 & 0.6 & +5.13 & 0. & 5 & 3986 & 0.512 & 3.2 & 1.4 & 53.5 & -11.2 & 0 & NM \\
\hline 118 & & 191 & 000641.59 & -302119.5 & 12.54 & 0.7 & -25.50 & 0.2 & 4 & 358 & 0.608 & 1.4 & 3.0 & 16.1 & -9.9 & 0 & NM \\
\hline 119 & & 195 & 000652.03 & -302308.0 & 11.82 & 0. & +3.85 & 0.21 & 5 & 3991 & 0.771 & 4.2 & 1.3 & 9.9 & -40.4 & 0 & NM \\
\hline 120 & 263 & 194 & 000652.80 & -301946.1 & 10.86 & 0. & -7.43 & 1. & 1 & & & 20.6 & 2.4 & 25.5 & 4.5 & 0 & NM \\
\hline 122 & 266 & 201 & 000702.20 & -302117.9 & 11.75 & 0. & +5.31 & 0.27 & 5 & 3991 & 0.485 & 13.2 & 0.8 & 20.9 & 2.5 & 90 & \\
\hline 125 & 282 & 217 & 000734.19 & -301714.1 & 10.59 & 0.44 & -0.53 & 1.00 & 4 & 3994 & 0.031 & 22.0 & 2.8 & 22.2 & 3.4 & 96 & \\
\hline 130 & 309 & 241 & 000900.63 & -295555.6 & 10.07 & 0.46 & +20.92 & 0.33 & 5 & 3989 & 0.054 & 7.5 & 0.9 & 70.3 & 41.6 & 0 & NM \\
\hline 330 & 2 & & 235653.03 & -295139.1 & 11.76 & 0.57 & +3.88 & 0.39 & 2 & 397 & 0.888 & 10.5 & 1.4 & 21.1 & 2.7 & 92 & \\
\hline 331 & 40 & & 235828.40 & -294908.3 & 11.91 & 0.65 & +5.72 & 1.06 & 2 & 397 & 0.008 & 12.1 & 2.0 & 21.4 & 3.3 & 95 & SB? \\
\hline 332 & 60 & & 235902.32 & -301317.2 & 10.87 & 0.50 & +5.33 & 1.32 & 6 & 3971 & 0.026 & 30.9 & 5.2 & 22.2 & 3.5 & 96 & $\mathrm{dbl}$ \\
\hline 333 & 73 & & 235944.56 & -304450.4 & 11.22 & & +29.95 & 0.20 & 5 & 3990 & 0.840 & 6.0 & 1.0 & -13.8 & -65.4 & 0 & NM \\
\hline
\end{tabular}


Table 1. continued.

\begin{tabular}{|c|c|c|c|c|c|c|c|c|c|c|c|c|c|c|c|c|c|}
\hline $\begin{array}{l}\text { No } \\
(1)\end{array}$ & $\begin{array}{l}\text { SA } \\
(2)\end{array}$ & $\begin{array}{l}\mathrm{ZS} \\
\text { (3) }\end{array}$ & $\begin{array}{l}\text { RA } \\
(4) \\
\end{array}$ & $\begin{array}{c}\text { Dec } \\
(5)\end{array}$ & $\begin{array}{c}V \\
(6) \\
\end{array}$ & $\begin{array}{c}B-V \\
(7)\end{array}$ & $\begin{array}{r}V_{\mathrm{r}} \\
(8) \\
\end{array}$ & $\begin{array}{c}\epsilon \\
(9) \\
\end{array}$ & $\begin{array}{r}N \\
(10) \\
\end{array}$ & $\begin{array}{r}\Delta T \\
(11) \\
\end{array}$ & $\begin{array}{r}P\left(\chi^{2}\right) \\
(12)\end{array}$ & $\begin{array}{c}V \sin i \\
(13)\end{array}$ & $\begin{array}{r}e v s \\
(14) \\
\end{array}$ & $\begin{array}{c}\mu_{\alpha} \cos \delta \\
(15)\end{array}$ & $\begin{array}{c}\mu_{\delta} \\
(16)\end{array}$ & $\begin{array}{r}\operatorname{Pr} \\
(17) \\
\end{array}$ & $\begin{array}{l}\text { Notes } \\
(18)\end{array}$ \\
\hline 334 & 107 & & 000108.42 & -283656.5 & 11.63 & 0.60 & +6.28 & 0.47 & 2 & 397 & 0.337 & 16.2 & 3.0 & 19.9 & 1.1 & 5 & \\
\hline 335 & 109 & & 000115.59 & -282935.6 & 11.29 & & +33.33 & 0.25 & 4 & 4407 & 0.570 & 4.3 & 1.8 & 22.6 & -2.8 & 0 & NM \\
\hline 336 & 147 & & 000232.91 & -305931.4 & 11.18 & 0.53 & +4.69 & 1.84 & 2 & 394 & 0.018 & 41.8 & 4.9 & 20.6 & 1.9 & 70 & \\
\hline 337 & 155 & & 000248.80 & -291842.2 & 12.33 & 0.76 & +6.12 & 0.32 & 2 & 396 & 0.279 & 3.7 & 2.1 & 20.5 & 3.6 & 81 & \\
\hline 338 & 181 & & 000338.51 & -28 3725.4 & 12.27 & 0.73 & +5.25 & 0.33 & 2 & 396 & 0.718 & 9.0 & 1.3 & 22.3 & -0.6 & 0 & M: \\
\hline 339 & 247 & & 000612.36 & -282834.1 & 10.58 & & +30.24 & 0.21 & 4 & 2586 & 0.743 & 5.3 & 1.2 & 12.0 & 0.3 & 0 & NM \\
\hline 340 & 250 & & 000617.78 & -291840.5 & 12.04 & 0.66 & +6.56 & 0.34 & 2 & 11 & 0.515 & 8.8 & 1.4 & 19.8 & 3.0 & 43 & \\
\hline 341 & 284 & & 000742.36 & -285532.5 & 11.12 & 0.54 & +8.40 & 0.19 & 5 & 3989 & 0.962 & 4.1 & 1.2 & -3.3 & -14.4 & 0 & NM \\
\hline 342 & 291 & & 000802.31 & -284940.8 & 11.70 & & +31.47 & 0.30 & 4 & 3979 & 0.703 & 11.0 & 1.1 & 15.8 & -16.9 & 0 & NM \\
\hline 343 & 320 & 246 & 000929.18 & -304245.3 & 10.75 & 0.50 & +4.02 & 0.23 & 5 & 3990 & 0.884 & 9.2 & 1.3 & 19.0 & 11.7 & 0 & NM \\
\hline 344 & 327 & & 000946.98 & -291343.3 & 9.98 & 0.55 & +6.35 & 0.17 & 5 & 3976 & 0.478 & 5.4 & 0.8 & -76.5 & -54.2 & 0 & NM \\
\hline 345 & & & 235903.98 & -300953.4 & 12.16 & 0.52 & +7.16 & 0.34 & 5 & 3990 & 0.171 & 4.4 & 2.0 & -9.5 & -10.4 & 0 & NM \\
\hline 346 & & & 235752.27 & -300518.0 & 12.16 & 0.70 & +4.70 & 0.39 & 2 & 397 & 0.732 & 12.5 & 1.3 & 22.3 & 2.5 & 93 & $\mathrm{dbl}$ \\
\hline 347 & & & 235918.33 & -30 1016.6 & 12.61 & 0.79 & +5.26 & 0.35 & 2 & 397 & 0.326 & 10.3 & 1.3 & 22.0 & 2.7 & 94 & \\
\hline 348 & & & 000325.04 & -30 2606.2 & 11.97 & 0.75 & +13.23 & 4.06 & 3 & 3637 & 0.000 & 0.5 & 4.0 & 22.3 & 3.1 & 95 & SB \\
\hline 349 & & & 235757.01 & -292140.0 & 12.88 & 0.91 & +4.89 & 0.37 & 2 & 397 & 0.578 & 8.1 & 1.7 & 22.4 & 3.9 & 90 & $\mathrm{dbl}$ \\
\hline 350 & & & 001308.26 & $\begin{array}{llll}-30 & 15 & 32.1\end{array}$ & 12.27 & 0.63 & +0.14 & 0.35 & 2 & 395 & 0.842 & 4.8 & 2.8 & 18.2 & 0.0 & 0 & NM \\
\hline 351 & & & 000114.00 & -292518.2 & 12.44 & 0.76 & +5.16 & 0.39 & 2 & 396 & 0.231 & 8.6 & 1.9 & 19.0 & 5.0 & 0 & M: \\
\hline 352 & & & 000319.80 & -29 2528.7 & 12.80 & 0.82 & +5.96 & 0.36 & 2 & 397 & 0.479 & 5.1 & 2.4 & 20.1 & 3.6 & 55 & \\
\hline 353 & & & 000400.84 & -292646.3 & 12.58 & 0.79 & +6.03 & 0.62 & 2 & 396 & 0.064 & 8.1 & 1.5 & 19.5 & 2.1 & 9 & \\
\hline 354 & & & 000434.85 & -291520.0 & 12.86 & 0.86 & +6.02 & 0.37 & 2 & 397 & 0.466 & 5.1 & 2.4 & 20.9 & 2.9 & 88 & \\
\hline 355 & & & 001027.92 & -312156.2 & 12.19 & 0.67 & +26.16 & 0.34 & 2 & 395 & 0.757 & 2.6 & 2.5 & 20.5 & -0.9 & 0 & NM \\
\hline 356 & & & 001042.32 & -292611.6 & 12.51 & 0.74 & +10.22 & 0.33 & 2 & 395 & 0.825 & 3.1 & 2.2 & 22.6 & 3.9 & 90 & \\
\hline 357 & & & 000037.05 & -30 1154.7 & 10.87 & 0.50 & +40.61 & 0.44 & 1 & & & 2.6 & 0.5 & 5.8 & -24.6 & 0 & NM \\
\hline 358 & & & 000653.69 & -283108.8 & 12.45 & 0.74 & +6.36 & 0.26 & 2 & 10 & 0.461 & 3.7 & 1.8 & 21.2 & -0.2 & 0 & M: \\
\hline 359 & & & 00045 & -290910.9 & 12.75 & 0.98 & +6.39 & 0.58 & 2 & 9 & 0.468 & 22.7 & 2.3 & 21.3 & 1.4 & 52 & $\mathrm{dbl}$ \\
\hline 360 & & & 000733.71 & -312351.5 & 12.98 & 0.87 & +10.22 & 0.43 & 1 & & & 0.4 & 3.8 & 23.0 & 2.4 & 76 & \\
\hline 361 & & & 23561 & -294734.7 & 13.01 & 0.61 & -1.73 & 0.64 & 1 & & & 0.5 & 5. & 19.5 & 1.5 & 2 & NM \\
\hline 1013 & 89 & 13 & 000035 & -301121.1 & 12.16 & 0.82 & +5.33 & 0.49 & 5 & 3979 & 0.468 & 18.4 & 1.3 & 23.1 & -1.6 & 0 & $\mathrm{M}:, \mathrm{db}$ \\
\hline 1021 & & 21 & 000106.24 & -30 1446.1 & 13.35 & 0.88 & +64.08 & 0.58 & 1 & & & 2.9 & 3.6 & 16.3 & -4.6 & 0 & NM \\
\hline 1025 & & 25 & 000110.31 & -303624.8 & 12.83 & 0.96 & +13.38 & 0.54 & 1 & & & 6.4 & 4.0 & 33.2 & -27.4 & 0 & NM \\
\hline 1029 & 115 & 29 & 000128.67 & $\begin{array}{lll}-30 & 12 & 21.0\end{array}$ & 10.86 & 0.46 & +4.21 & 1.00 & 5 & 3979 & 0.981 & 39.8 & 4.0 & 21.0 & 2.5 & 92 & \\
\hline 1031 & & 31 & 000133.20 & -30 1259.7 & 12.47 & 0.74 & +5.93 & 0.26 & 4 & 3979 & 0.799 & 7.9 & 1.1 & 21.7 & 3.0 & 95 & $\mathrm{dbl}$ \\
\hline 1033 & & 33 & 000129.45 & -30 2424.4 & 14.02 & 1.03 & -4.21 & 0.77 & 1 & & & 3.7 & 5.0 & -12.6 & -15.1 & 0 & NM \\
\hline 1034 & & 34 & 000149.50 & -301512.0 & 12.93 & 0.69 & -40.38 & 0.69 & 2 & 2 & 0.055 & 3.0 & 2.4 & 22.4 & 0.4 & 3 & NM \\
\hline 1044 & & 44 & 000214.70 & -294904.1 & 13.50 & 0.95 & +6.20 & 0.28 & 4 & 3981 & 0.984 & 8.0 & 1.2 & 22.1 & 3.0 & 92 & \\
\hline 1045 & & 45 & 00218.56 & -295108.4 & 12.95 & 0.85 & +6.02 & 0.26 & 4 & 3980 & 0.708 & 6.0 & 1.5 & 22.1 & 2.0 & 84 & \\
\hline 1050 & 142 & 50 & 021 & -30 3227.2 & 12.20 & 0.70 & +6.27 & 0.32 & 4 & 3979 & 0.225 & 9.5 & 1.0 & 21.8 & 3.2 & 96 & \\
\hline 1054 & & 54 & 000228.19 & -300443.4 & 13.03 & 1.00 & +4.24 & 0.58 & 1 & & & 13.5 & 1.8 & 21.8 & 2.5 & 90 & $\mathrm{dbl}$ \\
\hline 1058 & & 58 & 0146.47 & -294638.8 & 12.37 & 0.74 & +5.42 & 0.47 & 4 & 3978 & 0.044 & 11.6 & 1.0 & 20.8 & 2.5 & 86 & $\mathrm{dbl}$ \\
\hline 1062 & & 62 & 000235.46 & -300701.9 & 12.56 & 0.77 & +4.57 & 3.41 & 3 & 1788 & 0.000 & 5.0 & 2.2 & 21.2 & 2.9 & 93 & SB \\
\hline 1063 & 151 & 63 & 000236.14 & -302551.0 & 11.87 & 0.60 & -3.16 & 0.25 & 4 & 3979 & 0.414 & 3.7 & 1.8 & 18.3 & -11.0 & 0 & NM \\
\hline 1070 & & 70 & 000248.23 & -294634.8 & 13.80 & 1.02 & +5.09 & 0.36 & 3 & 3982 & 0.292 & 7.0 & 2. & 20.9 & 3.2 & 86 & \\
\hline 1075 & & 75 & 000300.28 & -300321.6 & 12.87 & 0.92 & +5.06 & 0.41 & 4 & 3290 & 0.151 & 7.8 & $1 .(\mathrm{r}$ & 21.5 & 3.9 & 91 & $\mathrm{dbl}$ \\
\hline 1076 & & 76 & 000256.39 & -300444.8 & 12.44 & 0.87 & +5.60 & 11.86 & 2 & 10 & 0.000 & 8.4 & 1.4 & 21.6 & 3.2 & 95 & SB2 \\
\hline 1078 & & 78 & 000302.88 & -30 2016.4 & 13.09 & 0.98 & +12.43 & 0.95 & 1 & & & 5.1 & 4.6 & 18.6 & -17.1 & 0 & NM \\
\hline 1093 & & 93 & 000324.68 & -295514.7 & 14.23 & 1.14 & +7.22 & 0.54 & 1 & & & 6.3 & 0.0 & 22.2 & 2.4 & 83 & \\
\hline 1095 & & 95 & 000316.49 & -295847.4 & 12.42 & 0.92 & +6.83 & 0.53 & 2 & 11 & 0.125 & 10.2 & 2.8 & 20.1 & 2.7 & 60 & $\mathrm{dbl}$ \\
\hline 1100 & & 100 & 000327.33 & -30 2648.8 & 12.55 & 0.79 & +3.74 & 3.42 & 4 & 3627 & 0.000 & 12.7 & 1.9 & 22.4 & 3.1 & 94 & SB \\
\hline 1111 & & 111 & 000407.54 & -300635.2 & 12.74 & 0.87 & +32.03 & 0.60 & 1 & & & 3.1 & 3.7 & 11.0 & -12.7 & 0 & NM \\
\hline 1112 & & 112 & 000404.02 & -295826.4 & 13.06 & 0.86 & +4.96 & 0.33 & 3 & 3978 & 0.584 & 6.8 & 1.6 & 22.6 & 4.0 & 86 & \\
\hline 1113 & & 113 & 000407.74 & -295300.5 & 13.05 & 0.92 & -22.53 & 0.53 & 1 & & & 3.4 & 0.0 & 29.8 & -20.5 & 0 & NM \\
\hline 1118 & & 118 & 000417.49 & -30 2342.4 & 13.07 & 1.01 & +6.19 & 0.43 & 1 & & & 4.9 & 2.6 & 21.8 & 2.7 & 92 & dbl \\
\hline 1122 & & 122 & 000425.26 & -30 2539.7 & 12.59 & 0.79 & +4.48 & 0.21 & 4 & 3982 & 0.469 & 0.6 & 0.0 & -7.6 & -17.5 & 0 & NM \\
\hline 1124 & & 124 & 000421.73 & -294530.1 & 13.87 & 1.07 & +5.92 & 0.32 & 3 & 3975 & 0.607 & 5.9 & 1.7 & 21.9 & 3.0 & 90 & \\
\hline 1135 & & 135 & 000443.55 & -294104.8 & 13.86 & 1.00 & +5.37 & 0.31 & 3 & 3982 & 0.494 & 2.6 & 2. & 21.7 & 3.7 & 89 & \\
\hline 1141 & & 141 & 000508.24 & -30 2942.1 & 12.00 & 0.67 & +6.00 & 0.22 & 4 & 3979 & 0.661 & 4.9 & 1. & 20.4 & 3.8 & 74 & \\
\hline 1147 & & 147 & 000517.56 & -294657.3 & 13.57 & 0.95 & +5.88 & 0.31 & 3 & 3978 & 0.932 & 4.8 & 2.0 & 23.0 & 3.1 & 81 & \\
\hline 1148 & & 148 & 0514.40 & -295423.7 & 12.40 & 0.85 & -5.18 & 24.70 & 2 & 10 & 0.000 & 8.6 & 1.5 & 22.8 & 4.0 & 87 & SB \\
\hline 1151 & & 151 & 0520.15 & $\begin{array}{llll}-30 & 25 & 22.0\end{array}$ & 13.08 & 0.91 & +19.07 & 0.43 & 2 & 8 & 0.973 & 2.5 & 3. & 9.4 & -7.9 & 0 & NM \\
\hline 1153 & & 153 & 0523.55 & $\begin{array}{lll}-30 & 23 & 31.9\end{array}$ & 12.39 & 0.73 & -12.81 & 0.41 & 3 & 3981 & 0.123 & 5.0 & 1.8 & 50.2 & 18.8 & 0 & NM \\
\hline 1154 & & 154 & 0531.57 & -30 2051.6 & 13.47 & 0.98 & +7.91 & 0.33 & 3 & 3982 & 0.859 & 6.6 & 1.8 & 22.0 & 2.2 & 84 & $\mathrm{dbl}$ \\
\hline 1161 & & 161 & 000526.86 & -295120.5 & 12.67 & 0.79 & +2.22 & 0.49 & 2 & 344 & 0.192 & 4.0 & 3.0 & 23.3 & 3.9 & 75 & \\
\hline 1165 & 233 & 165 & 000535.53 & -29 5706.4 & 12.49 & 0.88 & +11.77 & 20.02 & 2 & 10 & 0.000 & 5.0 & 1.7 & 21.0 & 3.8 & 89 & SB \\
\hline
\end{tabular}


Table 1. continued.

\begin{tabular}{|c|c|c|c|c|c|c|c|c|c|c|c|c|c|c|c|c|c|}
\hline $\begin{array}{l}\text { No } \\
(1)\end{array}$ & $\begin{array}{l}\text { SA } \\
(2)\end{array}$ & $\begin{array}{l}\mathrm{ZS} \\
(3)\end{array}$ & $\begin{array}{l}\text { RA } \\
(4)\end{array}$ & $\begin{array}{l}\text { Dec } \\
(5)\end{array}$ & $\begin{array}{c}V \\
(6)\end{array}$ & $\begin{array}{c}B-V \\
(7)\end{array}$ & $\begin{array}{l}V_{\mathrm{r}} \\
(8)\end{array}$ & $\begin{array}{c}\epsilon \\
(9)\end{array}$ & $\begin{array}{r}N \\
(10)\end{array}$ & $\begin{array}{r}\Delta T \\
(11)\end{array}$ & $\begin{array}{c}P\left(\chi^{2}\right) \\
(12)\end{array}$ & $\begin{array}{c}V \sin i \\
(13)\end{array}$ & $\begin{array}{r}e v s \\
(14)\end{array}$ & $\begin{array}{c}\mu_{\alpha} \cos \delta \\
\text { (15) }\end{array}$ & $\begin{array}{c}\mu_{\delta} \\
(16)\end{array}$ & $\begin{array}{r}\operatorname{Pr} \\
(17)\end{array}$ & $\begin{array}{l}\text { Notes } \\
(18)\end{array}$ \\
\hline 1170 & & 170 & 000554.73 & -300625.8 & 12.12 & 0.71 & +5.76 & 0.32 & 4 & 3981 & 0.282 & 9.7 & 1.1 & 22.0 & 2.5 & 94 & \\
\hline 1177 & 245 & 177 & 98 & 29374 & 13.32 & & 75.72 & 1.21 & 1 & & & 24.4 & 6.0 & & 1.4 & 0 & NM \\
\hline 1180 & 253 & 180 & 062 & 3030 & 5 & & -1.13 & 0. & 4 & 2158 & 0.331 & 4.4 & 1.4 & 38.6 & -7.2 & 0 & \\
\hline 1181 & & 181 & 0061 & -3022 & 13.05 & & +2.95 & 1.84 & 1 & & & 5.9 & 7.7 & 22.1 & 4.1 & 88 & \\
\hline 1187 & & 187 & 0063 & -29531 & 14.11 & $1 .($ & +3.85 & 0.61 & 1 & & & 10.9 & 4.5 & 4.9 & -4.1 & 0 & \\
\hline 1196 & & 196 & 0065 & -30311 & 11.46 & 0.68 & +19.83 & 0.21 & 4 & 3982 & 0.613 & 1.7 & 1.8 & -52.1 & -71. & 0 & $\mathrm{~N}$ \\
\hline 1203 & & 203 & 000713.70 & -294825.1 & 13.62 & 0.93 & -11.38 & 0.61 & 1 & & & 7.4 & 4.3 & 67.5 & 2.7 & 0 & NM \\
\hline 1213 & & 213 & 000728.63 & $\begin{array}{llll}-30 & 13 & 32.7\end{array}$ & 12.47 & 0.76 & +21.18 & 0.35 & 2 & 9 & 0.669 & 4.0 & 3.2 & 46.1 & 2.9 & 0 & $\mathrm{~N}$ \\
\hline 1214 & 281 & 214 & 000738.22 & -29 5029.7 & 11.34 & 0.60 & +3.54 & 7.60 & 4 & 345 & 0.000 & 6.2 & 1.7 & 9.5 & -15.1 & 0 & $\mathrm{NM}, \mathrm{SB}$ \\
\hline 1218 & & 218 & 000749.70 & -3029 & 2.36 & 0.72 & +5.63 & 0.26 & 4 & 3981 & 0.416 & 8.0 & 1.2 & 20.8 & 2.5 & 86 & \\
\hline 1222 & & 222 & 8.03 & -301 & 2.98 & 0.94 & +49.83 & 0. & 1 & & & 0.4 & 0.0 & 11.9 & -1.5 & 0 & $\mathrm{~N}$ \\
\hline 1223 & & 223 & .07 & -30 & 8 & 1 & 59. & & 2 & 5 & 0.303 & 3.1 & 2.9 & 51.7 & -25.9 & 0 & \\
\hline 1227 & & 227 & & & 9 & & 30.25 & & 1 & & & 3.5 & 0.0 & 3.3 & -18.8 & 0 & $\mathrm{~N}$ \\
\hline 1228 & & 228 & & & & 0. & +31.66 & & 1 & & & 10.1 & 2.5 & -35.8 & -31.6 & 0 & \\
\hline 1231 & 302 & 231 & & & & & & & 3 & 345 & 0.984 & 3.6 & 2.3 & & -0.3 & & $\mathrm{~N}$ \\
\hline 1234 & & 234 & & & & & -14 & & 1 & & & 2. & 0 & 12.4 & -18.1 & & \\
\hline 1239 & & 239 & & & & & +9 . & & & 1810 & 0.0 & 1. & & & 1. & 0 & \\
\hline 1243 & & 243 & & & & 0. & -1 . & & & 3981 & 0.0 & 5.1 & 2 & & 3. & 92 & \\
\hline 1251 & 323 & 251 & & -300725.4 & 11.16 & 0. & -1.03 & 12.23 & 4 & 3981 & 0.000 & 2.9 & 2. & 51.9 & -14.0 & 0 & NM, SB \\
\hline 1258 & 332 & 258 & 000955.68 & -300058.2 & 11.42 & 0.56 & -10.44 & 0.22 & 5 & 3982 & 0.987 & 5.5 & 1.2 & 20.7 & -12.5 & 0 & NM \\
\hline
\end{tabular}

Table content: (1) numbering system used in WEBDA and based on Westerlund et al. (1988) list, up to \#130, from \#330 to 361 new candidate stars. Numbers from 1013 to 1258 are based on de Epstein \& Epstein (1985) list (No + 1000), (2) numbers from the Selected Area 140 (Brück 1935), and (3) numbers of de Epstein \& Epstein (1985), (4) and (5) J2000 right ascension and declination from Platais (2008), (6) and (7) photoelectric $V$ and $B-V$ data from Westerlund et al. (1988) for stars with numbers $<131$, and new CCD data from James et al. (2008), for stars with numbers higher than $300,(8)$ mean radial velocities $\left(V_{\mathrm{r}}\right),(9)$ errors $(\epsilon)\left[\mathrm{km} \mathrm{s}^{-1}\right],(10)$ number of measurements $(N),(11)$ the time interval covered by the observations $(\Delta T)$, and (12) the probability $P\left(\chi^{2}\right)$ that the scatter is due to random noise, (13) projected rotational velocities $(V$ sin $i)$ derived from CORAVEL correlation functions, and (14) their error (evs), (15) and (16) $\mu_{\alpha} \cos \delta$ and $\mu_{\delta}$ in mas $\mathrm{yr}^{-1}$, and (17) membership probabilities from Platais (2008), (18) remarks on (non)membership (NM - non-member, M: - member from RV and photometry, but not from proper motion) and duplicity (SB - spectroscopic binary, dbl - member according to RV and $\mu$, and appears to be a photometric double star).

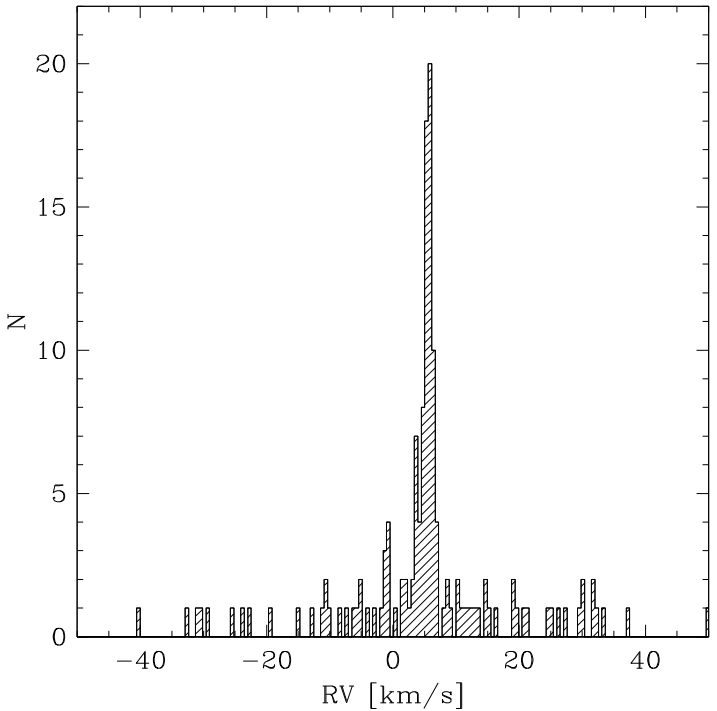

Fig. 1. Histogram of mean radial velocities for the present sample of pre-selected possible cluster members.

cluster mean and fall exactly on the MS in the colour-magnitude diagram. A fifth star (1013) appears to be a photometric double with the RV matching the cluster's mean radial velocity. Examination of the proper motions for these stars shows that the $\mu_{\alpha} \cos \delta$ component corresponds to the cluster mean value, while the $\mu_{\delta}$ component differs significantly from the mean value and is responsible for the null probability. We suspect that the $\mu_{\delta}$ component of the proper motion could be biased because of

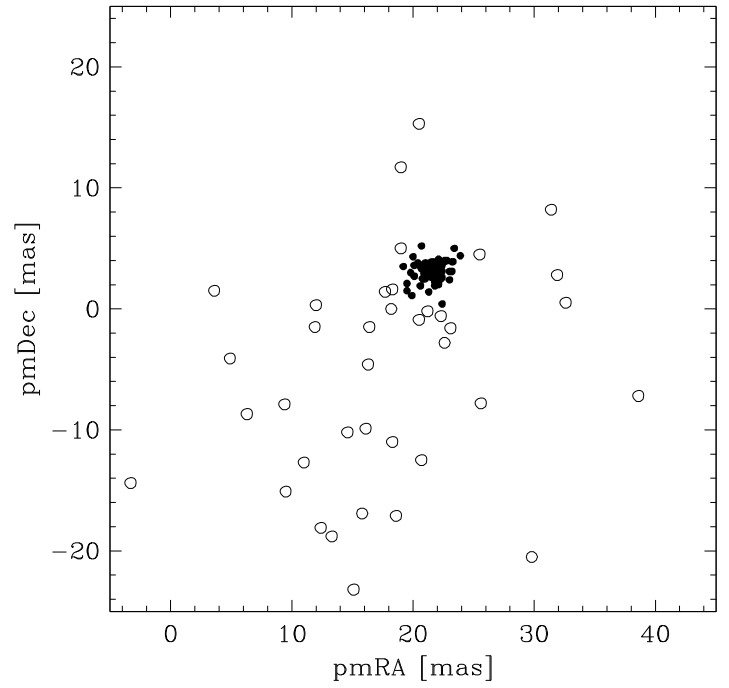

Fig. 2. Vector-point diagram of new proper motions. Symbols: bold dots - members including those marked as (M:); open circles non-members.

possible image overlaps which may occur in the presence of secondary images produced by the wire-grating on astrometric photographic plates and CCD frames. These five stars are flagged "M:" in Table 1 and are considered as likely members.

Conversely, three other stars $(21,361$, and 1034) have low non-zero membership probabilities but they clearly are nonmembers according to their radial velocities. In addition all three 


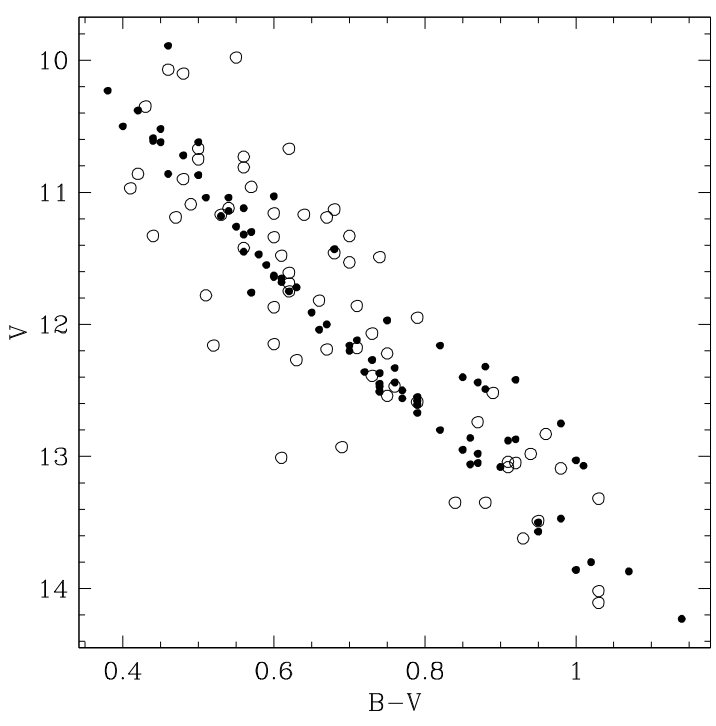

Fig. 3. Colour-magnitude diagram of all stars observed in the current programme. Several non-member stars are located near the main sequence, indicating an ambiguity of the photometric criterion alone for selecting the cluster members. Bold dots: members; open circles: non-members.

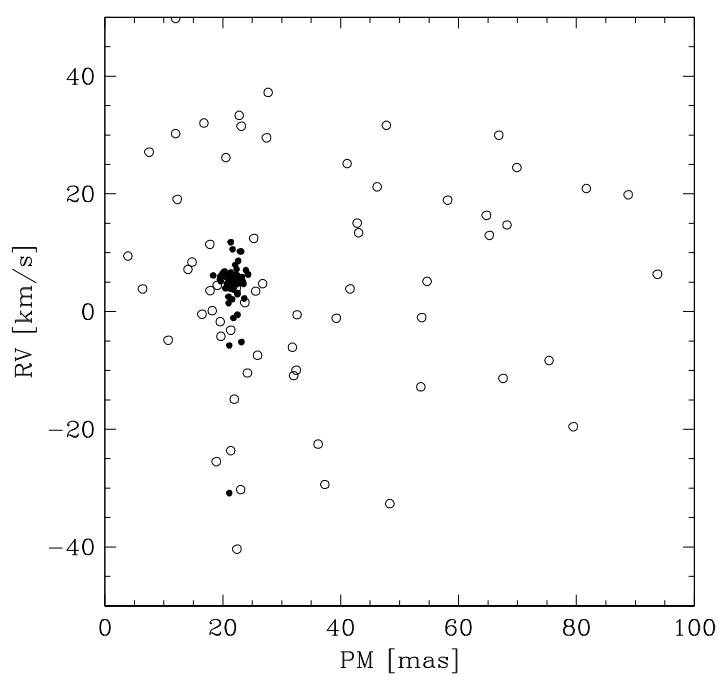

Fig. 4. Proper motions versus radial velocities. Bold dots: members; open circles: non-members.

are located well below the MS in the colour-magnitude diagram (Fig. 6).

\subsection{Photometry}

Our original sample was based on photometrically selected main-sequence stars, using just lower accuracy photographic photometry. It is obvious from Fig. 3 that $V, B-V$ photometry alone is not efficient in selecting the true cluster members in the field of Blanco 1. A significant fraction of stars in the original sample fall within the MS location, but their radial velocities and/or proper motions indicate that they are merely field stars. We therefore need to rely on kinematic and/or astrometric criteria to obtain the final cluster-member selection.

According to our photometric criterion, stars were considered to be cluster members if they are located on the main sequence $( \pm 0.1 \mathrm{mag})$ or between the MS (single star locus) and the binary ridge, 0.75 mag brighter. The new CCD data allow us to

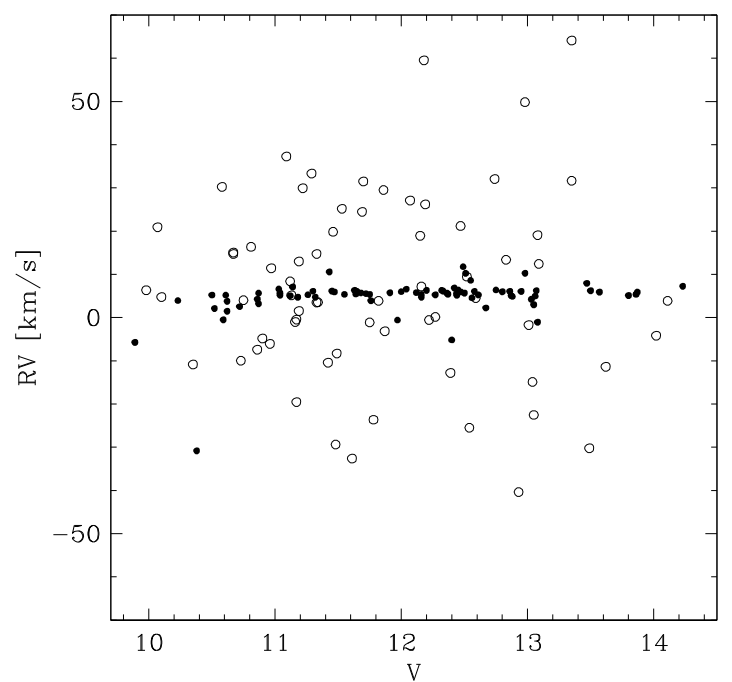

Fig. 5. Distribution of radial velocities as a function of $V$ magnitude. The symbols are the same as in Fig. 4.

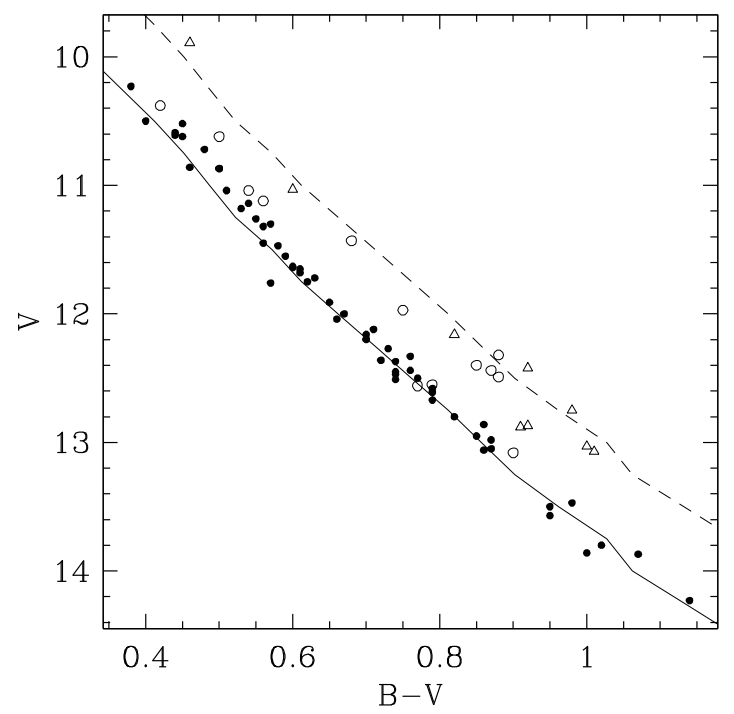

Fig. 6. Colour-magnitude diagram of the cluster members. Symbols are: bold dots: members; open circles: spectroscopic binaries; open triangles: photometric binaries. The solid curve is the ZAMS and the dashed one, the upper binary ridge.

define more tightly the locus of single stars. Seven stars located above the MS in the $(V, B-V)$ diagram (Fig. 6) by more than 0.3 mag are identified as binaries (Table 1: SB in Col. 18) from the photometry and also by their radial velocities $(19,58,95$, $348,1076,1148,1165)$. These binaries also have a high propermotion membership probability.

Eight additional stars $(20,37,349,359,1054,1075,1095$, 1118) are located well above the MS in both the $(V, B-V)$ (Fig. 6) and $(V, V-I)$ (Fig. 7) diagrams with $\delta V>0.5$ mag. However, they are not detected as spectroscopic binaries. Five stars (332, $346,1031,1058$ and 1181 ) are located about $0.3-0.4$ mag above the single-star sequence in the $(V, V-I)$ diagram only. Most likely some of the red secondaries show up in $I$ bandpass, but not in $B$ bandpass and are probably of late $\mathrm{K}$ or $\mathrm{M}$ type, with $\Delta V=V_{B}-V_{A}>4 \mathrm{mag}$. Their constant radial velocities and proper motions fully support their cluster membership. They are considered to be photometric double stars and labelled "dbl" in Table 1 . These stars should be re-observed in radial velocity or 


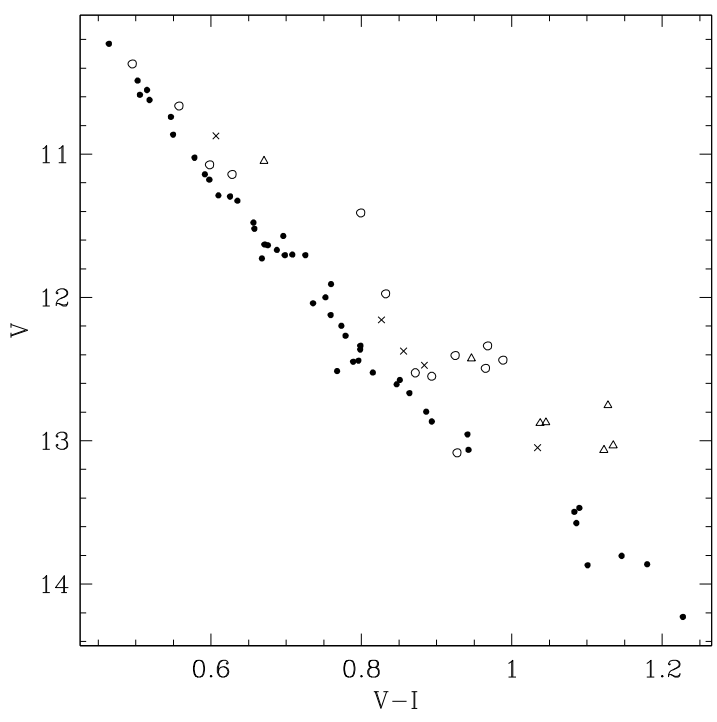

Fig. 7. Colour-magnitude diagram of the cluster members. Symbols are: bold dots: members; open circles: spectroscopic binaries; open triangles: photometric binaries from $B V$ data; crosses: photometric binaries from $V I$ data.

with high spatial-resolution imaging to test whether they are indeed long-period, wide-separation binary systems.

Conversely, several stars lie very close to the MS, but have radial velocities and proper motions differing from the cluster's mean values. Thus, the stars 39, 45, 54, 118, 341, 1151, 1153, $1180,1223,1258$ are declared cluster non-members.

\subsection{Membership summary}

The membership-selection principles based on radial velocities and proper motions are illustrated in a plot displaying the total proper motions $\mu=\sqrt{(} \mu_{\alpha}^{2} \cos \delta+\mu_{\delta}^{2}$ ) (PM in Fig. 4) versus radial velocities. The scatter present in the direction of the radialvelocity axis at $\mu \sim+22$, results from the presence of spectroscopic binaries, because their mean velocity may differ from the cluster systemic velocity.

Another way to display the results of a cluster member selection based on kinematic data is to plot the radial velocity as a function of $V$ magnitude (Fig. 5). The relationship is very tight; the observable scatter is due to spectroscopic binaries the membership of which is confirmed by proper motions.

An analysis of radial velocities shows that 12 stars could be considered as candidate cluster members, however, they ought to be rejected because of incompatible photometry and/or proper motions. A few stars have proper motions marginally compatible with the cluster membership although otherwise they seem to be true cluster members. The bottom line is that all three criteria are fundamentally important in a reliable determination of cluster membership. As the result, we distinguish single cluster members (a), spectroscopic binary members (b), photometric binary members (c), probable members (d), uncertain members (e), photometric non-members (f), and field stars (g). These classes of cluster membership, including a brief description, are listed below.

a. Single members. Single stars with radial velocities within $2 \mathrm{~km} \mathrm{~s}^{-1}$ of the cluster mean radial velocity and with high propermotion membership probabilities, lying close to the main sequence, and not known to be a RV-variable $\left(P\left(\chi^{2}\right)>0.005\right)$.
There are 45 such stars: $28,38,52,53,56,60,63,64,74,79$, 80, 85, 89, 91, 99, 110, 113, 122, 332, 334, 336, 337, 340, 346, $347,352,353,354,356,1029,1031,1044,1045,1050,1058$, 1070, 1093, 1112, 1124, 1135, 1141, 1147, 1154, 1170, 1218.

b. Spectroscopic binary members. Stars within the MS location having high-proper membership probabilities, but are radial-velocity variant, i.e., $P\left(\chi^{2}\right)=0.000$. A total of 14 stars are flagged as SBs: 2 SB2 (68 and 1076), 9 SB1 (57, 70, 95, $348,1062,1100,1148,1165,1243)$ and 3 SB? $(19,47$, and 331). Further radial velocities of star 57 are necessary to confirm its membership, which is supported by proper motion and photometry.

c. Photometric binary members. Stars with consistent radial velocities close to the cluster's mean, high proper-motion membership probabilities and a position in colour-magnitude diagram close to the binary ridge, indicating that they should have a companion. A total of 13 objects are considered to be "dbl". Among them, eight come from the $(V, B-V)$ diagram: 20, 37, $349,359,1054,1075,1095,1118$ and five from the $(V, V-I)$ diagram: $332,346,1031,1058$, and 1181 .

In several cases, the suspicion of binarity is also supported by the low value of $P\left(\chi^{2}\right)(<0.030)$ and the fact that the radial velocities differ by a few $\mathrm{km} \mathrm{s}^{-1}$ from the cluster mean velocity, i.e. $\Delta V_{\mathrm{r}}<5 \mathrm{~km} \mathrm{~s}^{-1}$.

d. Probable members. Stars which are located on the singlestar main sequence and have radial velocities close to the cluster mean, but the $\mu_{\delta}$ components of the proper motion are slightly deviant (biased) from the mean motion: 108, 338, 351, 358, 1013. Star 1013 would also be a candidate photometric double star (dbl).

e. Probable non-members from proper motions. Stars with radial velocities within $3 \sigma$ from the mean velocity but having zero formal proper-motion membership probability: 8, 92, 117, 119, 341, 343, 344, 345, 1122, 1187, 1214, 1239.

f. Photometric non-members. Star 330 satisfies both kinematic criteria but is located below the main sequence. If its photometry is correct, this could be an astrophysically interesting star (WD companion?).

g. Non-members. All stars with radial velocities outside the range $+2<V_{\mathrm{r}}<+10 \mathrm{~km} \mathrm{~s}^{-1}$ and with a zero propermotion membership probability are listed as non-members. In five cases, the radial velocities found in the literature confirm the CORAVEL values, implying that these are constantvelocity field-star interlopers in the Blanco 1 field (in parenthesis CORAVEL and the literature radial velocities in $\mathrm{km} \mathrm{s}^{-1}$, respectively): $1151(19.1,18.4), 1153(-12.8,-13.6), 1180$ $(-1.1,-1.5), 1228(31.7,32.7)$, and $1234(-14.9,-16.3)$.

\section{Results}

\subsection{Mean proper motion}

We computed the mean absolute proper motions from three different catalogues by using the list of class $a$ members. The Southern Proper Motion (SPM) programme (Platais et al. 1998) 
yields $\mu_{\alpha} \cos \delta=17.90 \pm 0.21, \mu_{\delta}=1.65 \pm 0.29$ mas $\mathrm{yr}^{-1}$ from 31 stars. This is an independent absolute proper motion for Blanco 1 using background galaxies as an astrometric reference frame. Two other widely used catalogues, TYCHO-2 and UCAC2, directly rely upon Hipparcos positions and proper motions and, hence, cannot be considered to be independent. For the sake of completeness, we provide the absolute proper motions of Blanco 1 in the system of these catalogues as well: $\mu_{\alpha} \cos \delta=$ $17.23 \pm 0.83, \mu_{\delta}=2.59 \pm 0.64 \mathrm{mas} \mathrm{yr}^{-1}$ (UCAC2, from 24 stars); $\mu_{\alpha} \cos \delta=18.67 \pm 1.10, \mu_{\delta}=2.35 \pm 0.74{\text { mas } \mathrm{yr}^{-1}}^{-1}$ (TCHO-2, from 16 stars).

These values are close to the Hipparcos mean absolute proper motion for Blanco 1: $\mu_{\alpha} \cos \delta=+19.15 \pm 0.50$ and

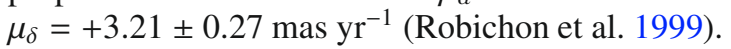

\subsection{Mean cluster velocity}

The mean radial velocity of Blanco 1 , based on 49 constantvelocity stars, is $+5.53 \pm 0.11 \mathrm{~km} \mathrm{~s}^{-1}$ (s.e.) and $0.82 \mathrm{~km} \mathrm{~s}^{-1}$ (rms). Stars 58, 74, 125, 356, and 360 were removed from the computation by the application of the $2 \sigma$ criterion during the second iteration. Our value is close to that reported by Jeffries \& James (1999), $+5.0 \pm 0.2 \mathrm{~km} \mathrm{~s}^{-1}$.

We note that Gonzalez \& Levato (2006) report a mean radial velocity ${ }^{1}$ for Blanco 1 equal to $+6.8 \pm 0.2 \mathrm{~km} \mathrm{~s}^{-1}$, in fair agreement with our mean velocity, although there is a $\simeq 1 \mathrm{~km} \mathrm{~s}^{-1}$ zero-point offset between their study and ours.

\subsection{Cluster space velocity}

By adopting the apparent distance modulus equal to $m-M=7.00$ and colour excess $E(B-V)=0.012$ deduced from the value of the interstellar polarization at the location of the cluster (Berdyugin et al. 2004), and using Hipparcos absolute proper motion, the total spatial velocity of Blanco 1 is $+23.4 \mathrm{~km} \mathrm{~s}^{-1}$. The corresponding components of the space velocity are: $U=-20.3, V=$ -6.5 , and $W=-9.7 \mathrm{~km} \mathrm{~s}^{-1}$. They are in good agreement with the values published by Makarov (2007), $U=-22.6, V=-7.0$, and $W=-8.2 \mathrm{~km} \mathrm{~s}^{-1}$.

Blanco 1 has the smallest space velocity of any young stellar group, which explains the difficulty to isolate members from proper motions or radial velocity only.

\subsection{Binary frequency}

Our radial velocity study of Blanco 1 resulted in the detection of 11 spectroscopic binaries among the cluster members: 2 SB2 and 9 SB1. They all have high proper-motion membership probabilities. Unfortunately, the available series of observations cover too short a time-span for us to calculate their orbital elements.

Three stars with low values of $P\left(\chi^{2}\right)$ and lying above the MS are suspected binaries (SB?). In addition, 13 stars lie close to the binary ridge and are considered as photometric doubles (dbl). Their kinematic parameters strongly favour cluster membership.

The fraction of spectroscopic binaries in Blanco 1 is about $20 \%(14 / 68)$. This value may be as high as $40 \%(27 / 68)$ if the suspected binaries, spectroscopic and especially photometric, are taken into account.

As was the case for the Pleiades (Mermilliod et al. 1992) and Praesepe (Mermilliod \& Mayor 1999), a number of spectroscopic binaries, namely 4 , were discovered among the photometric "single" stars. The fraction of binaries not detected by

\footnotetext{
1 The authors informed us about a mis-print in their paper.
}

the photometric analysis of $(V, B-V)$ colour-magnitude diagram may be at the level of $31 \%$ (Blanco 1) to 66\% (Praesepe). The use of $(V-I)$ indices permitted the detection of five more $\mathrm{K}$-dwarf binaries because the relative flux of a dM secondary is higher in the $I$ band than in the $V$ band.

\subsection{Colour-magnitude diagram}

The $(V, B-V)$ colour-magnitude diagram (Fig. 6) resulting from the cluster member selection based on three criteria (photometry, RVs and PMs) shows a well-defined main sequence and a number of binaries. The position of the MS from Mermilliod (1981) corresponds to a colour excess of $E(B-V)=0.012$ and an apparent distance modulus of $m-M=7.00$. The upper curve indicates the location of maximum displacement of equalcomponent binaries at 0.75 mag brighter than the MS. This is the best colour-magnitude diagram for Blanco 1 ever obtained in the magnitude range $10<V<14.5$, and it displays a very tight main sequence.

The $(V, V-I)$ diagram (Fig. 7) also shows a very tight main sequence. The separation of single stars and binaries is clearer because the redder secondaries have stronger effects on the $V-I$ colours than on the $B-V$ indices.

\subsection{Rotational velocities}

The distribution of projected rotational velocities, $V \sin i$, (Fig. 8), shows that the Blanco 1 members rotate faster than the field stars. The sequence displays characteristics similar to those of the Pleiades (Queloz et al. 1998), i.e. a rapid decrease of velocities around an F5 spectral type and a "plateau" for the latertype stars, the level of which is different from that defined by the field-star rotational velocities. It is clear that the late-F, $G$ and early-K stars of Blanco 1 must still loose a considerable fraction of their angular momentum to match the mass-dependent mean velocity of their (presumably older) field-star counterparts. A similar trend is also clearly visible in the plot showing the cumulative functions (Fig. 9) for the members and non-members.

The distributions of projected rotational velocities in Blanco 1 and the Pleiades are similar (Fig. 10). The cumulative distribution of the $V \sin i$ (Fig. 11) for stars in the colour interval $0.40<B-V<1.00$ confirms the similarity of the rotational velocities between Blanco 1 and the Pleiades. A nearly identical pattern of Li depletion in both open clusters (Jeffries \& James 1999) further strengthens the assumption that the ages of both clusters must be similar (Ford et al. 2005).

\subsection{Stability radius}

The mass of Blanco 1 was computed by summing the mass of known and probable members. The 11 stars brighter than $V=$ 9.5 produce a mass of $24 M_{\odot}$, the 68 stars observed with Coravel reach a mass of $76 M_{\odot}$, including the secondaries of the photometric binaries. We also took into account the probable members found by Moraux et al. (2007): 60 stars with $M \sim 0.50$ and 60 stars with $M \sim 0.30 M_{\odot}$. The total is $\sim 160 M_{\odot}$.

The stability radius can be computed with the relation $r_{\mathrm{t}} \sim$ $1.46\left(M_{\mathrm{cl}}\right)^{1 / 3}$. With $M_{\mathrm{cl}}=160 M_{\odot}, r_{\mathrm{t}}$ is equal to $7.90 \mathrm{pc}$. At a distance of $250 \mathrm{pc}$, the apparent radius is equal to 1.8 . The radius of our field, 1.5 , is slightly smaller than the stability radius, and therefore, additional members may be found at larger distances from the cluster centre. 


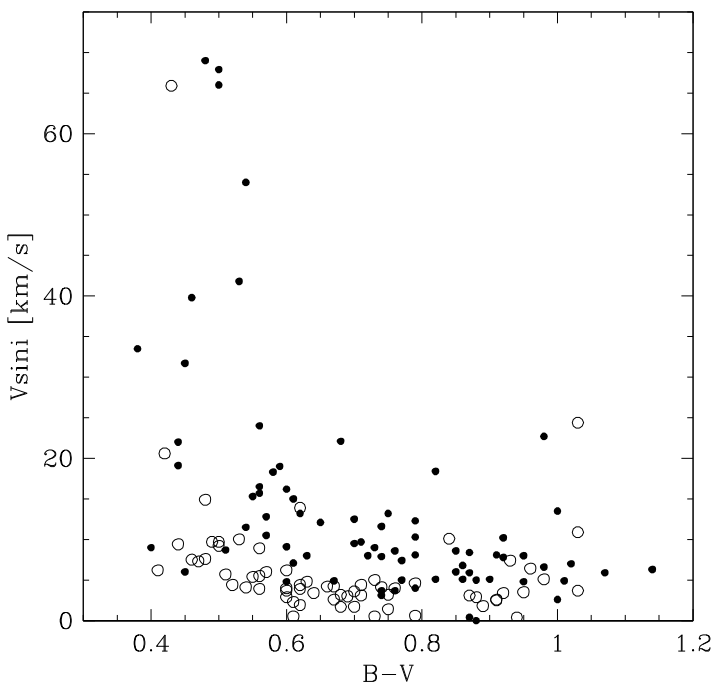

Fig. 8. Distribution of rotational velocities. On average, Blanco 1 cluster members (bold dots) rotate faster than the field stars (open circles).

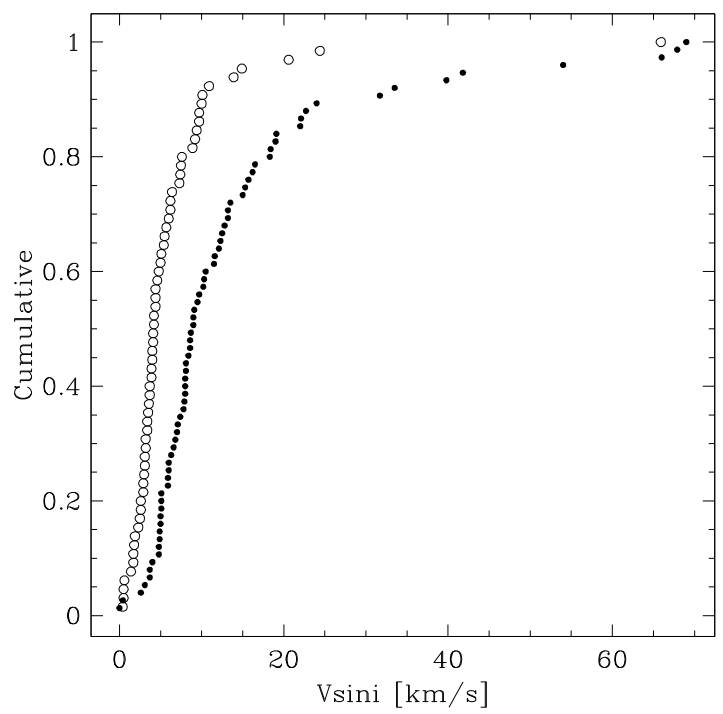

Fig. 9. Cumulative distribution of rotational velocities for cluster members (bold dots) and field stars (open circles).

\section{Conclusions}

The membership of 68 stars in the open cluster Blanco 1 in the range $10<V<14.2$ was confirmed using radial velocities, new proper motions and UBVI CCD photometry. We discovered 14 spectroscopic binaries among the members and additional 13 stars appear to be photometric binaries in the colour-magnitude diagrams. Four spectroscopic binaries (31\%) lie precisely on the main sequence and could not be detected by analysing the $V, B-V$ colour-magnitude diagram only.

Several Blanco 1 stars located on the main sequence have kinematic data incompatible with cluster membership. A few stars with high proper-motion membership probability appear to be field stars according to their radial velocities and location in the colour-magnitude diagram. This study strongly advocates the necessity of using all three kinematic, astrometric and photometric criteria to determine reliable membership for the rather poorly-populated Blanco 1 cluster, even though it lies at a high Galactic latitude and is expected to suffer from little field-star contamination.

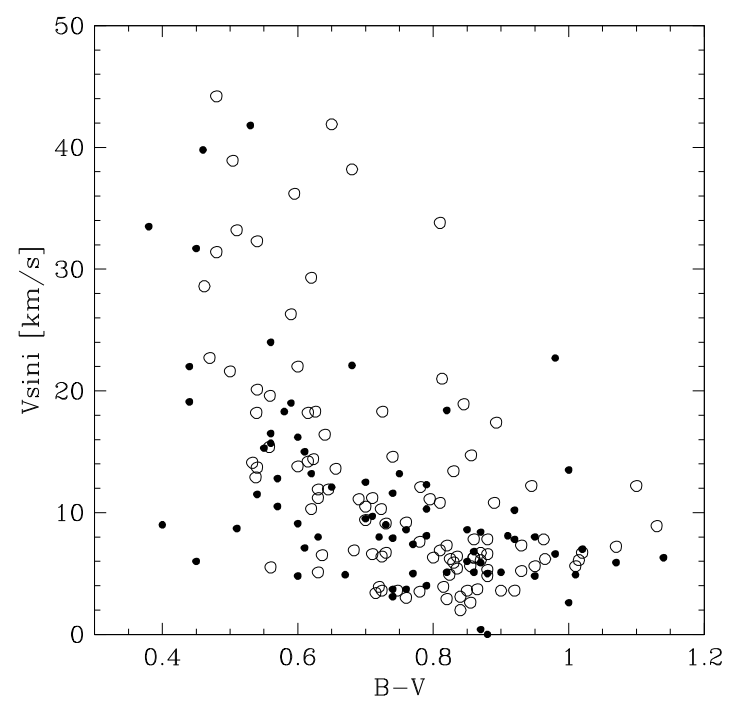

Fig. 10. Comparison of the $V \sin i$ distributions for Blanco 1 (bold dots) and the $100 \mathrm{Myr}$ Pleiades cluster (open circles).

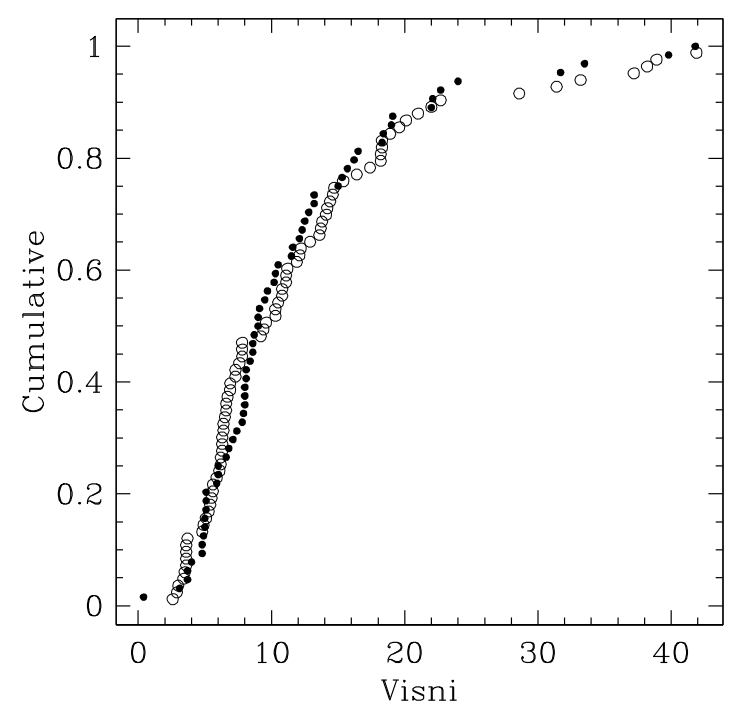

Fig. 11. Comparison of the $V \sin i$ cumulative distributions for Blanco 1 (bold dots) and the $100 \mathrm{Myr}$ Pleiades cluster (open circles) for stars with $0.40<B-V<1.00$.

On average, the solar-type cluster members rotate faster than the field dwarfs and evolved stars. The distribution of their projected rotational velocities is similar to that of the Pleiades. Finally, the paucity of X-ray luminosity data limits a reliable age-activity-rotation analysis, although we note that this magnetic-activity indicator seems to correlate with $V \sin i$.

Unfortunately, the present sample suffers from the piecemeal membership history of Blanco 1 and was limited by the extant cluster database at the time the observing programme started, and by the somewhat limited capabilities of the CORAVEL spectrograph on the $1.54 \mathrm{~m}$ Danish telescope. New astrometric and photometric studies in preparation (Platais et al. 2008; James et al. 2008) will provide a far more complete set of probable members to study the physical characteristics of this interesting cluster.

Although the upper main sequence of Blanco 1 is poorly populated, which makes any age determination by isochrone fitting difficult, the F-G spectral interval appears to be reasonably populated. Kinematic data are required to screen the lists 
of candidate members by Pillitteri et al. (2005) and Moraux et al. (2007) probing the dK-dM spectral range including brown dwarfs.

It has been firmly established that Blanco 1 is a bona fide open cluster, even though it is widely spread out on the sky ( $\simeq 3$ degrees). Whether it is stable against Galactic tidal forces remains to be proved by obtaining a more complete census of cluster members in terms of the on-sky spatial distribution, completeness and depth to which new members can be detected.

Acknowledgements. We thank Danilo Castillo, Kathy Vieira, and Terry Girard for observations and prompt reductions of the astrometric CCD data obtained at El Leoncito, Argentina. I. Platais gratefully acknowledges support from the National Science Foundation through grant AST 04-06689 to Johns Hopkins University. We also thank the referee, Dr R. D. Jeffries, for his valuable suggestions. The UBVI CCD observations were obtained at the SMARTS $1.0 \mathrm{~m}$ telescope at the Cerro Tololo Interamerican Observatory, to whose staff and Director we are most grateful. D. J. James and P. A. Cargile acknowledge support from a National Science Foundation grant AST-0349075 to Vanderbilt University. J.C. Mermilliod acknowledges research grants from the Swiss National Funds (FNRS).

\section{References}

Baranne, A., Mayor, M., \& Poncet, J.-L. 1979, Vistas Astron., 23, 279

Baranne, A., Queloz, D., Mayor, M., et al. 1996, A\&AS, 119, 373

Benz, W., \& Mayor, M. 1984, A\&A, 138, 183

Berdyugin, A., Piirola, V., \& Teerikorpi, P. 2004, A\&A, 424, 873

Brück, H. 1935, Publ. Astrophys. Obs. Potsdam, 28, 1

Casetti-Dinescu, D. I., Girard, T. M., Herrera, D., et al. 2007, AJ, 134, 195

de Epstein, A. E., \& Epstein, I. 1985, AJ, 90, 1211

Edvardsson, B., Pettersson, B., Kharrazi, M., \& Westerlund, B. 1995, A\&A, 293, 75

Ford, A., Jeffries, R. D., \& Smalley, B. 2005, MNRAS, 364, 272

Gonzalez, J. F., \& Levato, H. 2006. Rev. Mex. Astron. Astrofis. Conf. Ser., 26, 171
Hawkins, G. W., \& Favata, F. 1998, A\&AS, 130, 1346

Herbig, G. H., \& Jones, B. F. 1981, AJ, 86, 1232

James, D. J., Deliyannis, C. P., Steinhauer, A., et al. 2008, AJ, in prep.

Jeffries, R. D., \& James, D. J. 1999, ApJ, 511, 218

Laidler, V. G., Greene, G. R., Ray, K., Evzerov, A., \& Lasker, B. M. 1994, BAAS, 26, 897

Landolt, A. U. 1992, AJ, 104, 340

Makarov, V. V. 2007, ApJS, 169, 105

Mermilliod, J.-C. 1981, A\&A, 97, 235

Mermilliod, J.-C., \& Mayor, M. 1999, A\&A, 352, 479

Mermilliod, J.-C., Rosvick, J. M., Duquennoy, A., \& Mayor, M. 1992, A\&A, 265,513

Mermilliod, J.-C., Udry, S., \& Mayor, M. 2008, in prep.

Micela, G., Sciortino, S., Favata, F., Pallavicini, R., \& Pye, J. 1999, A\&A, 344, 83

Moraux, E., Bouvier, J., Stauffer, J. R., Barrado y Navascués, D., \& Cuillandre, J.-C. 2007, A\&A, 471, 499

Panagi, P. M., O’Dell, M. A., Collier Cameron, A., \& Robinson, R. D. 1994, A\&A, 292, 439

Panagi, P. M., \& O’Dell, M. A. 1997, A\&AS, 191, 213

Pillitteri, I., Micela, G., Sciortino, S., \& Favata, F. 2003, A\&A, 399, 919

Pillitteri, I., Micela, G., Sciortino, S., Damiani, F., \& Harnden Jr., F. R. 2004, A\&A, 421, 175

Pillitteri, I., Micela, G., Reale, F., \& Sciortino, S. 2005, A\&A, 430, 155

Platais, I., Girard, T. M., Kozhurina-Platais, V., et al. 1998, AJ, 116, 2556

Platais, I., Girard, T. M., Vieira, K., et al. 2008, in prep.

Queloz, D., Allain, S., Mermilliod, J.-C., Bouvier, J., \& Mayor, M. 1998, A\&A, 35,183

Randich, S., Schmitt, J. H. M. M., Prosser, C. F. \& Stauffer, J. R. 1996, A\&A, 305,785

Robichon, N., Arenou, F., Mermilliod, J.-C., \& Turon, C. 1999, A\&A, 345, 471

Stauffer, J. R., Caillault, J.-P., Gagné, M., Prosser, C. F., \& Hartmann, L. W. 1994, ApJS, 91, 625

Udry, S., Mayor, M., \& Queloz, D. 1999, in Precise Stellar Radial Velocities, IAU Colloq., 170, ed. J. B. Hearnshaw, \& C. D. Scarfe, ASP Conf. Ser., 185, 367

Vasilevskis, A., Klemola, A., \& Preston, G. 1958, AJ, 63, 387

Westerlund, B. E., Garnier, R., Lundgren, K., Pettersson, B., \& Breysacher, J. 1988, A\&AS, 76, 101

Zacharias, N., Urban, S. E., Zacharias, M. I., et al. 2004, AJ, 127, 3043 